\title{
L'enseignement de la génétique humaine : analyse de neuf manuels scolaires français et tunisiens
}

\section{Mondher Abrougui et Pierre Clément}

\section{(2) OpenEdition \\ 1 Journals}

Édition électronique

URL : http://journals.openedition.org/trema/1995

DOI : 10.4000/trema.1995

ISSN : 2107-0997

Éditeur

Faculté d'Éducation de l'université de Montpellier

Édition imprimée

Date de publication : 1 septembre 1996

Pagination : 33-41

ISSN : 1167-315X

\section{Référence électronique}

Mondher Abrougui et Pierre Clément, « L'enseignement de la génétique humaine : analyse de neuf manuels scolaires français et tunisiens », Tréma [En ligne], 9-10 | 1996, mis en ligne le 11 septembre 2013, consulté le 30 avril 2019. URL : http://journals.openedition.org/trema/1995 ; DOI : 10.4000/ trema.1995

Ce document a été généré automatiquement le 30 avril 2019

Trema 


\title{
L'enseignement de la génétique humaine : analyse de neuf manuels scolaires français et tunisiens
}

\author{
Mondher Abrougui et Pierre Clément
}

\section{Introduction}

1 Nous avons choisi d'étudier la façon dont la génétique humaine est présente dans l'enseignement de la génétique à l'école (enseignement secondaire). Notre hypothèse de départ est que des exemples pris sur l'homme sont plus motivants pour les élèves, mais qu'en même temps ils abordent des questions complexes par rapport auxquelles le dit et le non-dit sont lourds d'enjeux éthiques et sociaux. La querelle de l'inné et de l'acquis, bien qu'elle nous semble aujourd'hui dépassée sur le plan scientifique (nous adhérons par exemple aux positions exprimées à cet égard par Stewart, 1993), reste suffisamment vivace pour que tout discours sur les déterminismes génétiques de comportements ou pathologies humaines s'inscrive dans un contexte difficile, car vite critiqué par les uns ou les autres. Notre premier souci a donc été de mettre sur pieds une méthode efficace d'analyse du contenu des manuels scolaires choisis. Une autre hypothèse au départ de notre travail était que la façon dont le programme de génétique s'appuie ou non sur des exemples humains, nous semblait différer en France et en Tunisie : il nous importait de savoir si cette impression était fondée, et en quoi.

2 Nous avons donc décidé d'analyser la place de la génétique humaine dans l'enseignement de la génétique en comparant 9 manuels scolaires de deux pays différents, la France et la Tunisie. Nous avons limité cette comparaison aux manuels utilisés en 1994/95. Ils correspondent aux programmes officiels de 1994, en France pour les niveaux de $3^{\text {e }}$, de $\mathrm{r}^{\text {res }} \mathrm{S}$ et Terminale $S$, en Tunisie pour les niveaux de $3^{e}$ (dernière année de collège tunisien et équivalent au niveau de la $4^{\mathrm{e}}$ française), $6^{\mathrm{e}}$ (équivalent au niveau de la $1^{\text {res }}$ française) et terminale sciences expérimentales (équivalent au niveau de la terminale $\mathrm{S}$ française). Les 
programmes officiels dans les collèges et lycées secondaires tunisiens se réfèrent tous au décret $\mathrm{N}^{\circ}$ 93-670 du 29 mars 1993. Ces différents manuels sont présentés dans le tableau 1 :

Présentation des manuels du secondaire traités.

\begin{tabular}{|c|c|c|c|c|c|c|}
\hline \multicolumn{4}{|c|}{ France } & \multicolumn{3}{|c|}{ Tunisie } \\
\hline Années & $\begin{array}{l}\text { Programmes officiels } \\
\text { arrêté du }\end{array}$ & Nathan & Bordas & Années & $\begin{array}{l}\text { Programmes } \\
\text { officiels }\end{array}$ & $\begin{array}{l}\text { même } \\
\text { livre }\end{array}$ \\
\hline $3^{e}$ & $\begin{array}{l}14 / 11 / 85 \text { B.O. No } 44 \text { du } \\
12 / 12 / 85 \text { et } N^{0} 12 \mathrm{du} \\
23 / 03 / 1989\end{array}$ & 1989 & 1989 & $3^{e}$ & $\begin{array}{l}\text { décret No } 93-670 \\
\text { du } 29 \text { mars } 1993\end{array}$ & 1989 \\
\hline $1^{\text {re }} \mathrm{S}$ & $\begin{array}{l}\text { 10/07/92 B.O. hors série } \\
\text { du 24/09/92 tome II }\end{array}$ & 1993 & 1993 & $6^{\circ} \mathrm{SE}$ & $\begin{array}{l}\text { décret No } 93-670 \\
\text { du } 29 \text { mars } 1993\end{array}$ & 1994 \\
\hline Term S & $\begin{array}{l}\text { 08/04/94 B.0 spécial } \\
\text { No } 6 \text { du 09/06/94 }\end{array}$ & 1994 & 1994 & $\begin{array}{l}7^{e} \mathrm{SE} \\
\text { (terminale) }\end{array}$ & $\begin{array}{l}\text { décret No } 93-670 \\
\text { du } 29 \text { mars } 1993\end{array}$ & 1994 \\
\hline
\end{tabular}

\section{Méthodologie d'analyse des manuels}

3 À travers l'analyse de 9 manuels scolaires, six français et trois tunisiens, nous avons recherché l'importance que pouvait avoir la génétique humaine dans l'enseignement général de la génétique. Pour ce faire, nous avons essayé de savoir, parmi 20 notions que nous avons retenues pour l'analyse des 9 manuels, lesquelles étaient ou non traitées et quelle place leur était accordée dans chacun des manuels.

4 Ces notions sont les suivantes:

- caractère

- phénotype

- génotype

- caryotype

- chromosome

- gène

- allèle

- locus

- mutation

- ADN

- polymorphisme

- unicité

- environnement

- génétique classique

- génétique moléculaire

- génie génétique

- pedigrée

- médecine

- éthique

- idéologie

Les 17 premières notions concernent tous les secteurs de la génétique, alors que les 3 dernières sont plus spécifiques à la génétique humaine. Cette distinction à été faite après une première lecture des 9 manuels analysés. En effet, dans aucun des manuels il n'est 
question de médecine ni d'éthique autre qu'humaine; quant à l'idéologie, elle traverse peut-être tous les débats sur la génétique, mais elle est particulièrement identifiable dans tout ce qui concerne la génétique humaine.

\section{Comparaison des manuels des deux pays}

6 L'approche de l'enseignement de la génétique dans les manuels français et les manuels tunisiens s'est effectivement révélée très différente. Dans les manuels français, la majorité des notions de génétique sont introduites et explicitées par des exemples de génétique humaine. Dans les manuels tunisiens, surtout ceux de terminale et de $6^{\mathrm{e}}$, l'approche de la génétique s'appuie sur des exemples non humains.

7 Nous allons détailler l'analyse pour les 20 notions sélectionnées, en les regroupant parfois.

\subsection{La notion de caractère}

Dans l'ensemble des manuels, français et tunisiens cette notion est polysémique et renvoie pour le cas de l'homme aussi bien à des caractères génétiques que phénolypiques ou socioculturels. Or une distinction permettrait dès la $3^{\text {e }}$ d'éviter un héréditarisme excessif, et clarifierait aussi les idées sur l'hérédité des caractères acquis, débat qui a longtemps dominé l'histoire de la génétique (Abrougui, 1995).

9 La notion de caractère (phénotypique) occupe une place privilégiée en génétique, car elle appartient au domaine du concret, que l'élève peut voir, contrairement aux concepts plus formels (gène, allèle, locus, $\mathrm{ADN}$ ) que l'élève doit imaginer.

10 Un type de raisonnement est particulièrement favorisé dans les manuels tunisiens. Dans celui de $3^{\mathrm{e}}$, en l'absence de support explicite de la transmission génétique (gène, chromosome), le caractère devient le support du raisonnement dans la compréhension de l'hérédité: le caractère phénotypique est subrepticement confondu avec le caractère génétique. Dans les manuels tunisiens de $6^{\mathrm{e}}$ et de terminale, où les explicitations utilisent pourtant d'autres supports ( $\mathrm{ADN}$, gène, chromosomes, allèles, phénotype...) c'est toujours un raisonnement au niveau du «caractère » qui est privilégié. Au point que, dans le manuel de terminale, le concept de caractère remplace même celui de phénotype, ce dernier n'étant aucune fois explicité dans tous les exemples traitant de génétique humaine. Or l'une des approches que peut justement favoriser la génétique humaine est cette distinction entre caractère phénotype et génotype (Abrougui, 1995). Cette distinction et la prise en considération d'un modèle génétique comme support de l'expression des caractères seraient ici les bienvenues pour lever cette ambiguité de l'hérédité des caractères phénotypes.

\subsection{Les concepts de phénotype et de génotype}

11 Ces deux concepts sont étroitement liés et dans tous les manuels le second définit le premier. Ainsi, le phénotype apparait être comme l'expression directe du génotype. Aucun des manuels ne prend en compte l'environnement pour lequel à un même génotype peuvent correspondre des phénotypes forts différents : la différenciation des cellules du corps humain en est une illustration claire. 
12 D'autre part, le concept de phénotype est souvent employé de la même façon que la notion de caractère, ainsi que nous l'avons déjà signalé : les remarques précédentes restent donc pertinentes ici, notamment sur le danger de suggérer alors une transmission directe du phénotype.

\subsection{Les concepts d'unicité et de polymorphisme} premier réfère à l'unité et le second au pluriel. Cependant, la distinction entre ces deux concepts n'est aucune fois clairement explicitée comme dépendante de deux registres d'interprétation différents. Le polymorphisme relève des composantes les plus élémentaires, alors que le concept d'unicité concerne l'ensemble, la résultante globale des niveaux précédents. pour permettre cette distinction de registre. Mais cette approche est toujours accompagnée d'une approche mathématique rendant encore plus difficile sa conceptualisation. D'autre part, il est important d'aborder parallèlement des sources de diversité autres que génétique, par exemple culturelles ou sociales (voir notre atelier), afin de permettre la compréhension d'une unicité à la fois génétique et non génétique : l'ensemble des paramètres qui contribuent à façonner un individu et à le différencier des autres est loin d'être réductible à des caractéristiques génétiques: les capacités d'apprentissage de chacun jouent un rôle essentiel dans cette différenciation individuelle, en s'insérant dans une histoire individuelle (et sociale) unique. Ceci n'a été envisagé par aucun des manuels.

Sur ces mêmes concepts une divergence apparaît dans les approches entre les manuels français et tunisiens :

- Les manuels français traitent de ces notions, à tous les niveaux $3^{\mathrm{e}}, 1^{\text {res }} \mathrm{S}$ et terminale $\mathrm{S}$; en $1^{\text {re }}$ $\mathrm{S}$ et en terminale $\mathrm{S}$, ces concepts, qui sont aussi en partie abordés à travers des exemples autres qu'humains, sont toujours introduits et approfondis par des exemples de génétique humaine (seul le manuel Nathan $1^{\text {re }} S$ traite l'unicité avec autant d'exemples humains que non-humains). Toutefois, la réponse à la question de l'unicité des autres êtres vivants reste alors implicite.

- Les manuels tunisiens traitent ces deux notions de façon plus hétérogène. Dans le manuel de $3^{\mathrm{e}}$, elles ne sont pas du tout abordées. Dans celui de $6^{\mathrm{e}}$, le concept d'unicité reste spécifique à l'homme et n'est pas du tout traité par une autre approche, alors que le concept de polymorphisme est introduit à travers des exemples non-humains mais est approfondi par les deux types d'approches génétiques, de façon équivalente. Dans le manuel tunisien de terminale c'est plutôt une approche opposée à celle des manuels français qui est privilégiée. En effet, l'unicité et le polymorphisme des autres êtres vivants semblent être la règle, et aucune fois ces concepts ne sont traités à travers des exemples humains.

\subsection{Concepts de caryotype et de chromosome}

Ces deux concepts sont amplement développés par tous les manuels, sauf celui de $3^{e}$ tunisien. La place qui leur est consacrée est cependant moins importante dans les manuels tunisiens que dans les français. Le chromosome en métaphase dans un caryotype est un élément visuel structurant car, à l'instar de ce que nous avons dit plus haut sur la

Tréma, 9-10 | 2010 
notion de caractère, il contrebalance l'abstraction de la génétique. Ce chromosome en $\mathrm{X}$ réifie, pour les auteurs de manuels, la notion de gène ; il est présenté comme une preuve matérielle de la localisation des déterminants génétiques. Toutefois, un support chromosomique ne s'oppose pas à lui tout seul à la conception de l'hérédité des caractères ou des phénotypes, car il pourrait tout aussi bien être le support de ces caractères ou phénotypes. De plus il risque de renforcer la conception d'une hérédité par mélange. En effet, au cours de l'interphase précédant toute division cellulaire, les chromosomes non encore individualisés semblent être dissous dans le nucléoplasme, et pourraient laisser croire à un mélange des déterminants génétiques. Toutefois, les représentations graphiques, très nombreuses dans les 9 manuels, en matérialisant sur les chromosomes les gènes, les allèles et leur locus peuvent permettre de déplacer le raisonnement initialement pris au niveau du caractère ou du phénotype vers un raisonnement au niveau des gènes, ou allèles d'un même gène. Dans les manuels scolaires, l'immense majorité des chromosomes sont représentées en métaphase: soit dans des caryotypes, où ils sont déboublés (d'où la symétrie des bras du X), et où ils peuvent être associés par paire (associant deux X identiques); soit isolés du caryotype, sous forme d'un $\mathrm{X}$ simple qui devient leur représentation symbolique. Or les localisations de supports génétiques sur le chromosome sont schématisées sur un seul d'entre eux, ce qui risque d'induire chez les élèves une confusion entre allèle (sur un chromosome) et gène (qui comporte toujours deux allèles, chacun d'eux étant sur l'un des deux chromosomes homologues), à l'exception des chromosomes sexuels (XY chez l'homme). D'autre part, les représentations graphiques matérialisant les gènes ou leur allèles sur les chromosomes, qui ne sont que des modèles explicatifs ; ils véhiculent une conception statique des gènes, alors que, depuis les travaux de Barbara Mc Clintock, nous connaissons leur plasticité et leur dynamique (Mayr, 1989).

\subsection{Concept de mutation}

Dans 6 manuels, le concept de mutation est très fortement approfondi par l'approche de la génétique humaine, et ceci même dans les 2 manuels tunisiens qui introduisent ce concept à travers une approche autre que l'approche humaine. Ce concept est explicitement abordé dans les manuels tunisiens de $6^{\mathrm{e}}$ et de terminale alors qu'il n'est pas du tout traité dans celui de $3^{\mathrm{e}}$ et que dans les manuels français de $3^{\mathrm{e}}$ et de $1^{\text {res }} \mathrm{Nathan}$, il est implicite. Le terme de mutation n'est clairement explicité clans les manuels français qu'en $1^{\text {res }}$ dans Bordas et en terminale S dans Bordas et Nathan. Le fait que ce concept soit très lié aux maladies génétiques humaines, au travers desquelles il est approfondi, lui donne un aspect très péjoratif. Une telle conception de la mutation peut s'avérer un obstacle dans sa prise en considération comme facteur positif de diversité dans l'évolution. D'autre part, la très faible prise en considération des mutations neutres restreint la diversité au seul effet phénotypique des mutations.

\section{6. « Environnement » (le déterminisme environnemental du phénotype)}

L'idée du déterminisme environnemental des phénotypes (résumée ici par le terme «Environnement »), est absente dans les manuels de $3^{\mathrm{e}}$ et de $1^{\text {res }}$ français et dans le 
manuel de terminale tunisien, elle n'est présente que dans seulement 4des 9manuels analysés. cependant différemment abordée selon les manuels et les niveaux scolaires. Dans les manuels français de $3^{e}$ et de terminale $S$ et dans le manuel tunisien de terminale, cette approche est très approfondie alors que dans les manuels français de $1^{\text {res }} \mathrm{S}$ et les manuels tunisiens de $6^{e}$ et de $3^{e}$ cette dimension apparaît moins importante. L'insistance qu'a l'enseignement français de la génétique à privilégier une approche médicale a été déjà remarquée par Rumelhard (1986). Toutefois, il est important de remarquer qu'à travers celle-ci sont introduites les idées de prédiction et de remédiation. Ces idées, dans le contexte héréditariste de ces chapitres de génétique, risquent d'être associées à des idéologies de normalisation, telles que l'eugénisme négatif, très prégnant dans les très nombreux écrits sur ce sujet dans la presse actuelle (Rose, 1995). Les manuels français commencent à associer certains aspects de la recherche médicale en génétique à une dimension éthique, en signalant notamment les implications sociales que de telles approches pourraient avoir (voir 3.8, Les questions idéologiques). Les manuels tunisiens, au contraire ne remettent aucune fois en cause les démarches scientifiques et n'associent jamais éthique et génétique humaine. La seule approche éthique présente, dans le manuel de terminale tunisien, concerne les inconvénients des mariages consanguins (problème qui reste actuel en Tunisie comme dans tout le pourtour méditerranéen).

\subsection{Les questions idéologiques}

24

Ces questions ne sont explicitement présentes ni dans le manuel Nathan de $1^{\text {res }}$ ni dans les manuels tunisiens de $3^{\text {e }}$ et de terminale. Dans les autres manuels, c'est particulièrement à travers le problème racial qu'elles sont abordées, surtout en complément des concepts d'unicité et de polymorphisme. Le manuel français Bordas terminale $\mathrm{S}$ (enseignement de spécialité) est le seul à faire clairement référence à des idéologies qui ont mené, depuis 1917, à l'eugénisme négatif.

Si l'on explore l'implicite des contenus traités, les analyses résumées dans les paragraphes précédents mettent en évidence un héréditarisme omni présent: le parti- 
pris pédagogique initial, visant à bien faire comprendre aux élèves le déterminisme du phénotype par le génotype, est devenu un parti-pris orientant le choix des exemples et des silences et faisant dès lors, peut-être à son insu, jouer au génome une fonction prédéterministe, qui n'est pas sans rappeler le pré-déterminisme divin qui prévalut durant les siècles précédents (Clément et al, 1980).

Mendel et les lois de l'évolution.

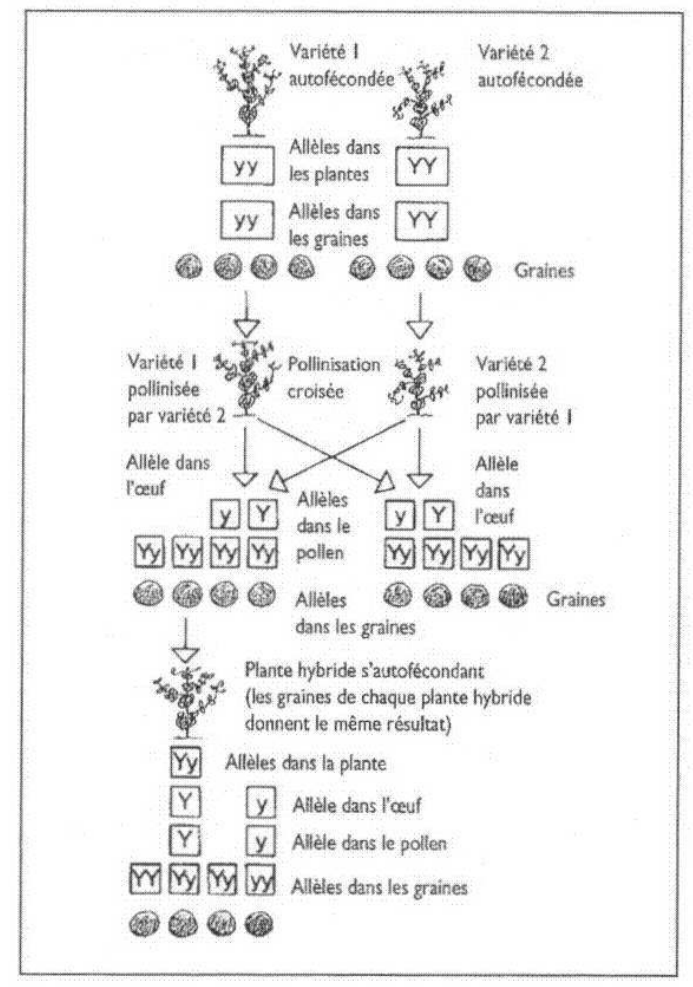

Illustration de la génétique Mendélienne : ici l'hybridation des plantes. Coll. : Passion des Sciences, L'Évolution, Paris, Gallimard, 1993.

\subsection{Génétique classique, génétique moléculaire et génie génétique}

Il s'agit des noyaux durs autour desquels s'articule l'ensemble de l'enseignement de la génétique.

- La génétique classique, reflet de l'approche mendélienne, est présente dans tous les manuels sauf dans les deux manuels-français de $1^{\text {res}}$. Dans les manuels de $3^{\mathrm{e}}$ français, qui introduisent ces notions uniquement par des exemples humains, et le manuel de $3^{\mathrm{e}}$ tunisien, qui introduit ces notions par des exemples autres que ceux de l'homme, les notions de génétique classique (dominance, récessivité, ...) sont approfondies à travers l'approche humaine. Alors que dans tous les manuels de terminale et le manuel tunisien de $6^{\mathrm{e}}, \mathrm{c}^{\prime}$ est à travers des approches ne référant pas au cas de l'homme que les notions de génétique classique sont approfondies.

- Les notions de génétique moléculaire sont introduites dès la $3^{\mathrm{e}}$ en France alors que clans les manuels tunisiens ces notions ne le sont que plus tard. Ces notions sont abordées d'une façon très approfondie dans les manuels de $1^{\text {re }} S$ française, de $6^{\mathrm{e}}$ tunisien et de terminale, classe où elles s'articulent fortement aux notions de génétique classique. L'approche humaine est privilégiée par les manuels français de Bordas $3^{\mathrm{e}}$, les deux manuels de terminale $\mathrm{S}$ et le manuel tunisien de terminale. Dans ce dernier, cette approche est même exclusive. 
Dans les autres manuels (Bordas $1^{\text {res }}$ et Nathan $3^{\text {e }}$ et $1^{\text {re }}$ ), les deux approches de génétique insistent autant sur ces notions. Le manuel de $6^{\mathrm{e}}$ tunisien est celui qui aborde les notions de génétique moléculaire en se référant le moins à l'homme. Au total, il apparaît donc clairement que les notions de génétique moléculaire difficiles à acquérir utilisent de nombreux exemples humains, car dans tous les manuels ces notions font référence au cas de l'homme.

- Les notions de génie génétique ne sont introduites qu'à partir de la terminale (sauf pour le manuel Bordas de $1^{\text {res }}$ qui les aborde, mais très superficiellement). Parmi ces 4 manuels (terminales S, Bordas $1^{\text {re }}$ et terminale tunisienne) seul le manuel de Nathan introduit et aborde ces notions à travers des cas d'exemples seulement humains. Les manuels de terminale $\mathrm{S}$ Bordas et tunisien, bien qu'ils introduisent tout deux ces notions à travers des exemples qui ne se réfèrent pas à l'homme, traitent aussi de cas d'exemples humains. Dans le manuel de terminale Bordas, ce sont les exemples humains qui approfondissent davantage ces notions. Le manuel Bordas $1^{\text {res}}$, quant à lui ne prend que des exemples autres qu'humains. Les notions de génie génétique apparaissent plus difficiles à introduire et nécessitent pour être appréhendées un support théorique important, qui n'est acquis qu'après l'enseignement de notions de biologie moléculaire. Le génie génétique n'est traité qu'une seule fois sans aucun exemple humain.

\subsection{Concept de pedigree}

Dans 5 des 9 manuels où le concept de pedigree est abordé (manuels de terminale $\mathrm{S}$ et tunisien ainsi que les manuels de $3^{\mathrm{e}}$ Bordas et tunisien), il n'est étudié qu'à travers l'exemple humain, sauf pour le cas du manuel de $3^{\mathrm{e}}$ tunisien, où ce concept est introduit par un exemple non humain. L'approche à travers les pedigrees de la génétique humaine reflète la réalité des investigations de recherche, tant historique qu'actuelle, qui privilégient cette démarche. Seulement, l'interprétation des pedigrees est toujours faite en termes mendéliens et ceci quels que soient les manuels utilisant cette approche. D'autre part aucun des manuels ne précise pourquoi ils ont choisi le cas de l'homme, ni n'explicite les difficultés de l'étude de l'hérédité humaine.

\section{Discussion et Conclusion}

Les manuels français et tunisiens présentent des différences qui sont les reflets de plusieurs contraintes. Celles imposées par les programmes, qui orientent les différents contenus d'enseignement. Celles de l'environnement socio-culturel, qui accompagnent toute élaboration de contenu. Cependant, pour les manuels français, malgré un même programme et un même environnement socio-culturel, quelques différences traduisent les choix des auteurs (cas des exemples privilégiés, importance de la prise en considération de facteur de l'environnement, etc.). L'ensemble de ces contraintes conduit à des choix de contenus plus ou moins explicités, et qui, parfois à leur insu, véhiculent certaines conceptions sur la génétique.

Une première conclusion concerne des différences liées aux niveaux de formulation et aux degrés d'explicitation. En effet, on constate que, dans les manuels tunisiens, moins de concepts et de notions sont abordés que dans les manuels français correspondants, particulièrement dans le manuel tunisien de $3^{\mathrm{e}}$. Il existe cependant quelques exceptions : au niveau du manuel tunisien de $\mathbf{6}^{\mathrm{e}}$ les notions de génétique classique sont abordées d'une 
façon très approfondie, alors que les manuels français ne développent cette approche qu'en classes de terminale, après quelques notions rapides dans les manuels de $3^{\mathrm{e}} \cdot \mathrm{Au}$ total, en considérant l'ensemble des manuels pour le même contenu d'enseignement de la génétique, il apparaît très clairement que le noyau central de cet enseignement est la génétique moléculaire; la génétique classique a une place moindre, puis le génie génétique.

D'autres constatations valent pour les 9 manuels analysés :

- Les éléments d'interprétation génétique tels que gènes, allèles, $\mathrm{ADN}$, bases azotées, sont abordés de façon à ne privilégier qu'un raisonnement de type linéaire causal, très admirablement mis en place par les effets des mutations sur les structures protéiques et, par voie de conséquence, sur les phénotypes et les caractères.

- Le très faible intérêt, si ce n'est parfois l'absence d'intérêt, pour les facteurs de l'environnement privilégie de façon implicite, en plus du raisonnenent causal linéaire, une idéologie héréditariste. Elle est par exemple présente dans les manuels quand ils laissent à penser que la machinerie moléculaire, en s'exprimant par les règles de transmission mendélienne, suit un programme déterminé dès la naissance et dirige l'expressions des caractères indépendamment du milieu dans lequel elle se déroule. Mais en plus, l'approche sur le polymorphisme et l'unicité, même si elle discrédite au passage les approches raciales, privilégie aussi ce déterminime génétique strict.

- Dans un tel contexte, l'importante approche médicale centrée sur l'identification de déterminismes génétiques, présente un double risque. D'une part, l'approche thérapeutique de remédiation couplée à un contexte héréditariste risque de renforcer des idées fatalistes sur la maladie. D'autre part, en insistant sur les approches préventives centrées sur les composantes génétiques des futurs individus, elle risque de cautionner une idéologie eugéniste, privilégiant une purification éthnique et visant une normalisation des populations humaines.

31 Or en médecine humaine, de nombreuses approches, tant curatives que préventives, centrent leur action sur la prise en considération des milieux d'expression, bien plus facile à contrôler. C'est le cas, particulièrement, de nombreux exemples du contrôle des régimes alimentaires pour certaines maladies souvent déterminées, pour une part, par des composantes génétiques, telles certaines formes de diabètes, les maladies métaboliques (phénylcétonurie), etc.

L'impact du comité national d'éthique, qui a pour fonction d'éviter ces dérives, commence à apparaître dans les manuels français, mais de telles considérations éthiques sont totalement absentes des manuels tunisiens. Dans ces derniers s'il est question d'éthique c'est par rapport au seul problème des mariages consanguins encore très importants dans ce pays.

Ce premier compte rendu d'une recherche en cours sur les contenus de 9 manuels de deux pays différents, nous a permis de montrer que plusieurs implicites accompagnent l'enseignement de la génétique, et notamment de la génétique humaine ; celle-ci occupe une place importante bien qu'elle soit utilisée de façon différente par les manuels français et tunisiens. Reliant étroitement les problèmes socioculturels et les connaissances en biologie, les exemples introduits par les manuels privilégient trop les seuls modèles explicatifs de déterminisme génétique, exprimant ainsi, à leur insu, des pensées héréditaristes, ouvrant implicitement au fatalisme génétique et à l'eugénisme négatif. 
Il est possible de comprendre la volonté des rédacteurs des programmes et des manuels, de prendre des exemples simples pour introduire les élèves aux notions fondamentales de la génétique. Mais c'est cette simplification / réduction qui, en privilégiant systématiquement des déterminismes génétiques simples, devient l'héréditarisme latent des manuels. D'autres exemples sont possibles, qui permettent d'introduire les concepts principaux de la génétique tout en présentant d'emblée la diversité des mécanismes par lesquels se construit un phénotype, ainsi que les divers enjeux (éthique et sociaux) de ces questions. Il y a là des différences fondamentales entre l'enseignement de la génétique humaine et celui des mathématiques ou de la physique.

\section{BIBLIOGRAPHIE}

ABROUGUI M., «Connaissances et idéologies dans l'histoire de la génétique humaine », in Actes J.I.E.S., №17, 1995, p. 267-272.

CLÉMENT P., BLAES N. \& LUCIANI A., « Le mythe tenace du <chromosome du crime>, encore appelé « chromosome de l'agressivité », in Raison Présente, №54, 1980, p. 109-127.

MAYR E., Histoire de la biologie. Diversité, évolution et hérédité, trad en français par M. Blanc, Paris,

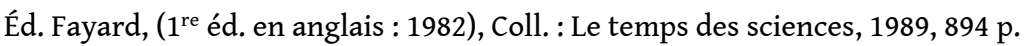

ROSE S., « À quand les gènes du bien et du mal ? », in Le Cahier Science de Libération, $\mathrm{N}^{\circ}:$ 21, Paris, Mars, 1995.

RUMELHARD G., La génétique et ses représentations dans l'enseignement, Berne, Peter Lang, 1986.

STEWARTJ., « Au delà de l'inné et de l'acquis », in Intellectica, Nº 16,Paris, Éd. CNRS, 1993. p. 151-174.

ZAZZO R., Le paradoxe des jumeaux, Paris, Stock/Laurence Pernoud, 1984, 242 p.

\section{RÉSUMÉS}

Ce texte présente l'analyse de la façon dont les manuels scolaires français et tunisiens traitent la génétique humaine dans le programme de génétique. Cette analyse identifie les contenus explicites ou implicites des manuels en fonction d'une vingtaine de notions scientifiques du champ de la génétique. Les ouvrages tunisiens explicitent moins que les français les enjeux éthiques et sociaux. Un implicite héréditarisme imprègne, cependant, l'ensemble des manuels étudiés.

This text presents an analysis of the way French and Tunisian biology schoolbooks treat the subject of human genetics within their respective official genetics curriculum. This analysis identifies the explicit and/or implicit contents of the schoolbooks on the basis of some twenty scientic concepts in the field of genetics. The Tunisian books are less explicit than the French ones, on the ethical and social stakes involved in the teaching of genetics. 
INDEX

Mots-clés : didactique, génétique, héréditarisme, manuel scolaire

Keywords : didactic, genetic, hereditarist standpoint, schoolbook

\section{AUTEURS}

MONDHER ABROUGUI

Equipe de recherche en didactique de la biologie, LIRDIHST, université Claude Bernard de Lyon

\section{PIERRE CLÉMENT}

Equipe de recherche en didactique de la biologie, LIRDIHST, université Claude Bernard de Lyon 